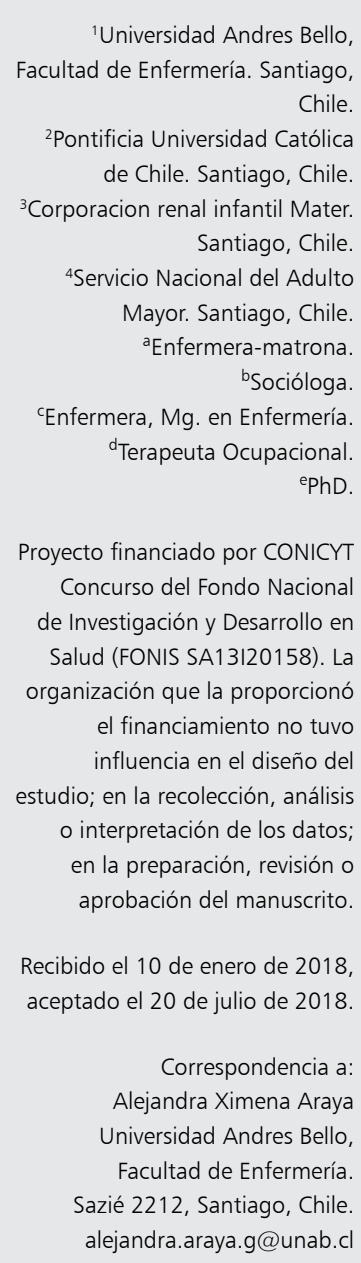

'Universidad Andres Bello, Facultad de Enfermería. Santiago, Chile.

${ }^{2}$ Pontificia Universidad Católica de Chile. Santiago, Chile. ${ }^{3}$ Corporacion renal infantil Mater. Santiago, Chile. ${ }^{4}$ Servicio Nacional del Adulto Mayor. Santiago, Chile. aEnfermera-matrona. ${ }^{\text {bSocióloga. }}$

`Enfermera, Mg. en Enfermería. ${ }^{\mathrm{d} T e r a p e u t a}$ Ocupacional. ePhD.

Proyecto financiado por CONICYT Concurso del Fondo Nacional de Investigación y Desarrollo en Salud (FONIS SA13I20158). La organización que la proporcionó el financiamiento no tuvo influencia en el diseño del estudio; en la recolección, análisis o interpretación de los datos; en la preparación, revisión o aprobación del manuscrito.

Recibido el 10 de enero de 2018, aceptado el 20 de julio de 2018.

Correspondencia a: Alejandra Ximena Araya Universidad Andres Bello, Facultad de Enfermería. Sazié 2212, Santiago, Chile. alejandra.araya.g@unab.cl

\section{Evaluación de la funcionalidad y fragilidad de las personas mayores asistentes a centros de día}

\author{
ALEJANDRA XIMENA ARAYA ${ }^{1, \mathrm{a}, \mathrm{e}}$, MARÍA SOLEDAD HERRERA ${ }^{2, \mathrm{~b}, \mathrm{e}}$, \\ EVELYN IRIARTE ${ }^{3, \mathrm{c}}$, ROMINA RIOJA ${ }^{4, \mathrm{~d}}$
}

\section{Changes in social psychological and functional variables among older people attending a day care center}

\begin{abstract}
Background: Older people attending day care centers improve their social activities, perception of quality of life and reduce their institutionalization rate and risk of mortality in the medium term. Aim: To evaluate the changes in the integral geriatric assessment and frailty of a group of older people attending a day care center in Santiago. Material and Methods: Health self-perception, integral geriatric assessment, Barthel scale, frailty indicators, mini-mental state scale, Yesavage depression score and a scale about solitude perception were evaluated in 35 participants aged $73 \pm 6$ years on admission and at discharge from a day care center for older people. Results: At discharge, significant improvements were observed for depression, perception of social support and physical frailty. However, no changes in functional status, cognitive function and perception of solitude were observed. Conclusions: Older people attending improve their perception of social support and decrease their frailty and depression scores.
\end{abstract}

(Rev Med Chile 2018; 146: 864-871)

Key words: Adult Day Care Centers; Frail Elderly; Geriatric Assessment.
E envejecimiento de la población representa uno de los grandes desafíos para los servicios socio-sanitarios a nivel mundial. La mayoría de los países industrializados han desarrollado una gama de servicios geriátricos destinados a promover la autonomía y calidad de vida de las personas mayores (PM); entre estos servicios geriátricos están los Centros de Día para Personas Mayores $(\mathrm{CDPM})^{1}$. En Chile, se han implementado desde el año 2013 a través de una política pública instaurada por el Servicio Nacional del Adulto Mayor, siendo un tipo de servicio gerontológico inédito en el país. Los CDPM realizan intervenciones para PM autovalentes/con dependencia leve ${ }^{2}$, que incluyen actividades de educación y promoción en salud, rehabilitación orientada hacia el mantenimiento de las capacidades funcionales, intervenciones psicosociales y actividades grupales, así como un descanso y apoyo a las familias cuidadoras ${ }^{3}$.

El Modelo del Envejecimiento Activo formulado en la Asamblea Mundial del Envejecimiento 2002 y promulgado por la OMS, se caracteriza por haberse convertido en el paradigma referencial para el desarrollo de políticas de países del mundo ${ }^{5}$. El desarrollo de servicios geriátricos de atención a PM para apoyar la permanencia en casa, junto con estrategias de prevención de la dependencia son dos pilares que este modelo establece ${ }^{5}$. Los CDPM juegan un papel importante en la prestación de servicios geriátricos para las PM frágiles, especialmente teniendo en cuenta los 
recursos disponibles para ellos, y se puede considerar como parte de la continuidad de cuidados a largo plazo ${ }^{6}$.

La evidencia internacional es consistente en señalar que la asistencia regular de las PM a CDPM se asocia con un aumento del funcionamiento psicosocial $^{2}$, parámetros cognitivos y afectivos ${ }^{4,7}$, autoestima ${ }^{8}$, calidad de vida ${ }^{9,10}$ y niveles de apoyo social ${ }^{11}$; junto con una disminución de sentimientos de soledad ${ }^{10}$, institucionalización ${ }^{13,14} \mathrm{y}$ del riesgo de mortalidad ${ }^{15}$. Sin embargo, no existe evidencia nacional en relación a la evaluación de las PM que asisten regularmente a este tipo de servicio geriátrico.

Este estudio contribuye a ampliar el conocimiento en dos aspectos: Primero, mide la fragilidad de las PM asistentes a los CDPM no centrándose exclusivamente en factores de funcionalidad y; segundo, amplia la evidencia científica desarrollada en países Latinoamericanos que tienen un contexto social y de atención geriátrica distinto a los países europeos o norteamericanos. El objetivo de este estudio fue evaluar los cambios en la valoración geriátrica integral y la fragilidad de un grupo de PM participantes de CDPM en Santiago al egreso de este servicio geriátrico.

\section{Material y Métodos}

\section{Diseño}

Analítico, longitudinal, prospectivo con dos aplicaciones, una al ingreso y otra al término de la participación en el CDPM, realizadas en una muestra al azar de 35 PM de un CDPM de la I. Municipalidad de Puente Alto en Santiago, Chile. Los criterios de selección fueron: PM de 60 años o más con dependencia leve según Barthel, sin diagnóstico de demencia. Hubo 3\% de rechazo a la participación; se perdieron 2 casos (inicialmente se evaluó a 37 personas).

\section{Recolección de datos}

Los participantes fueron entrevistados por el equipo de investigación a través de un método estandarizado para recolectar datos. La aplicación del cuestionario tomó cerca de 45 min para ser completado en dos instancias: al momento del ingreso al centro (Toma basal-T1) y a los 5 meses (Toma seguimiento-T2), previo egreso del centro. Esta toma final se hizo un mes antes del egreso del centro para evitar el sesgo de hacer una evaluación justo al momento del egreso.

\section{Medición de las variables e instrumentos}

Se midieron las siguientes variables:

a. Socio-demográficas: sexo, edad, estado civil, vive con pareja, tiene hijos y nivel de escolaridad.

b. Salud/enfermedad: auto-percepción de salud en escala de Likert de 4 puntos, número de enfermedades crónicas y hospitalizaciones auto-reportadas en el último año.

c. Valoración geriátrica integral (VGI): se midieron tanto variables de capacidad funcional y cognitiva, como de áreas psicológica y social como se describe a continuación:

c.1. Capacidad funcional. Se midió la capacidad de realización de las actividades de la vida diaria (AVD) mediante Escala de Barthel, con una puntuación de $0-100$, siendo la puntuación más alta las PM autovalentes ${ }^{16}$. Se usó la Escala de Lawton para medir las actividades instrumentales de la vida diaria (AIVD), donde 0 puntos representa dependencia y 8 puntos independencia total ${ }^{17}$.

c.2. Capacidad cognitiva y psicológica. Para medir el estado cognitivo se usó la prueba MMSE de Folstein para tamizaje de demencia. Esta tiene un rango total de puntuación del 0-30, donde puntajes más altos indican mejor función cognitiva. Los puntajes de la versión adaptada por edad y escolaridad fueron consideradas como deterioro cognitivo ${ }^{18}$. Los síntomas depresivos fueron medidos mediante la escala Yesavage, versión acortada de 5 ítems (Sí/No), donde la puntuación de 2 o más indica posible tamizaje de depresión ${ }^{19}$.

c.3. Área social. Fue medida a través del grado de soledad y apoyo social. El grado de soledad fue medido por el aislamiento social a través de la escala reducida de soledad de Hughes, basada en la original de Russell que tenía 20 preguntas. La escala tiene 3 ítems y 3 categorías de respuestas, con un rango de respuestas de 3 , dando un rango de 3 (sin aislamiento) a 9 (máximo aislamiento) y ha demostrado buenas propiedades psicométricas ${ }^{20}$, obteniendo un alpha 0,87 para este estudio. También se usó la Escala Multidimensional de Percepción de Apoyo 
Social (MSPSS) para medir apoyo social percibido en tres áreas: familia, amigos y otros significativos. Es una escala de 12 ítems con un Likert de 4 puntos, un rango de 12 (nula percepción de apoyo) a 48 (máximo apoyo) y que ha sido validada en población de $\mathrm{PM}$ chilenas ${ }^{21}$. En este estudio se reportó un alpha de 0,87 .

c.4. Síndrome de fragilidad. El instrumento Frailty Tilburg Indicator (TFI) fue utilizado para medir fragilidad total y en cada dominio: física (8 ítems), cognitiva (4 ítems) y social (3 ítems). El score se obtiene de la suma de sus preguntas, el rango va de 0-15, siendo frágil la PM que tenga 5 puntos o más. TFI ha mostrado buenas propiedades psicométricas $^{22-25}$ y tuvo un alpha 0,79 para esta población.

\section{Análisis de datos}

Análisis cuantitativo descriptivo con frecuencias, medidas de tendencia central y dispersión. Utilizando el programa estadístico IBM SPSS24, se calcularon test estadísticos para muestras pa- readas. En caso de las escalas que tienen nivel de medición escalar, se usaron test t Student para muestras dependientes. Como la mayoría de las escalas no tenía distribución normal y se comportaban más bien como variables ordinales, se calcularon también Pruebas de Rangos de Wilcoxon. Para las variables dicotómicas (escalas que tienen punto de corte y variables de fragilidad) se calculó Test $\chi^{2}$ de McNemar para muestras pareadas para variables dicotómicas.

\section{Ética}

Este estudio contó con la certificación Ética del Comité Ético Científico del Servicio de Salud Metropolitano Sur Oriente.

\section{Resultados}

\section{Descripción de la muestra}

Los resultados se muestran en la Tabla 1. El promedio de edad de la muestra fue de 73,31 \pm 6,11 años (mínimo 65-máximo 86 años). La mayoría eran mujeres $(82,9 \%) ; 31,4 \%$ solteros, $25,7 \%$ casados y la misma magnitud viudos; $34,4 \%$ vivía

Tabla1. Características sociodemográficas de la muestra en estudio

\begin{tabular}{|c|c|c|c|}
\hline \multicolumn{3}{|c|}{ 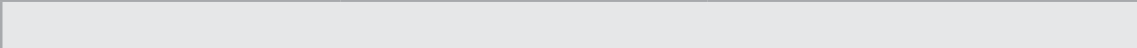 } & (N) \\
\hline \multicolumn{4}{|c|}{ Características sociodemográficas } \\
\hline \multicolumn{2}{|l|}{ Edad } & $\begin{array}{c}\text { Promedio }=73,31 \pm 6,11 \text { años } \\
\text { Rango: } 65-86 \text { años }\end{array}$ & $(35)$ \\
\hline \multicolumn{2}{|l|}{ Sexo femenino } & $82,9 \%$ & $(29)$ \\
\hline Estado civil & $\begin{array}{l}\text { Soltero (a) } \\
\text { Casado (a) } \\
\text { Separado (a) } \\
\text { Viudo (a) }\end{array}$ & $\begin{array}{l}31,4 \% \\
25,7 \% \\
17,1 \% \\
25,7 \%\end{array}$ & $\begin{array}{r}(11) \\
(9) \\
(6) \\
(9)\end{array}$ \\
\hline \multicolumn{2}{|l|}{ Vive con su pareja } & $34,3 \%$ & $(12)$ \\
\hline \multicolumn{2}{|l|}{ Tiene hijos } & $97,1 \%$ & $(34)$ \\
\hline Nivel educacional & $\begin{array}{l}\text { Nunca asistió a la escuela } \\
\text { Enseñanza básica } \\
\text { Enseñanza media } \\
\text { Enseñanza nivel técnico }\end{array}$ & $\begin{array}{r}5,7 \% \\
68,6 \% \\
22,9 \% \\
2,9 \%\end{array}$ & $\begin{array}{r}(2) \\
(24) \\
(8) \\
(1)\end{array}$ \\
\hline \multicolumn{4}{|l|}{ Características de salud } \\
\hline Autopercepción de salud & $\begin{array}{l}\text { Muy buena } \\
\text { Buena } \\
\text { Mala } \\
\text { Muy mala }\end{array}$ & $\begin{array}{l}8,8 \% \\
44,1 \% \\
26,5 \% \\
17,6 \%\end{array}$ & $\begin{array}{r}(3) \\
(15) \\
(9) \\
(6)\end{array}$ \\
\hline \multicolumn{2}{|c|}{ Con al menos una enfermedad crónica } & $94,3 \%$ & (33) \\
\hline \multicolumn{2}{|c|}{ Con hospitalización en el último año } & $5,7 \%$ & (2) \\
\hline
\end{tabular}


con pareja; $97,1 \%$ tenía hijos. Con respecto a su nivel educacional, 68,6\% reportó tener enseñanza básica (completa o incompleta), solo 2 participantes nunca asistieron a la escuela, pero sabían leer y escribir; el resto reportó enseñanza media o técnica incompleta. El 52,9\% PM evaluó su salud como muy buena/buena, mientras que el resto como mala/muy mala. Casi toda la muestra en estudio (94,3\%) tiene a lo menos una enfermedad crónica, siendo las más frecuentes enfermedades cardiovasculares y osteo-articulares; 2 casos estuvieron hospitalizados el último año.

En relación a la funcionalidad (Tablas 2 y 3), antes del ingreso al CDPM, el promedio del índice Barthel fue de 95,57, siendo clasificados un $37,1 \%$ como independientes y casi todo el resto como dependiente leve (hay un solo caso con valor 60, 2 casos con 90 y 19 con valor 95, siendo los accidentes de incontinencia urinaria los más mencionados). En relación a las AIVD, promedio antes del ingreso fue de 7,46, donde 77,1\% de los participantes obtuvieron el máximo puntaje del Îndice de Lawton. En cuanto al estado cognitivo, el MMSE detectó 3 personas con sugerencia de demencia después de aplicar el puntaje corregido por nivel educacional y edad; todo el resto de las PM obtuvo un puntaje normal (92\%). Se calcularon los test para muestras pareadas eliminando estos tres casos, comportándose de manera similar a los cómputos que los incluían, por lo que se tomó la decisión de no eliminarlos de la muestra.

Con respecto al tamizaje de depresión, la Escala de Yesavage tuvo un promedio de 2,17, clasificando a $62,9 \%$ de PM con un tamizaje sugerente de depre-

Tabla 2. Funcionalidad y fragilidad de las personas mayores asistentes al Centro de Día $(\mathbf{n}=\mathbf{3 5})$

\begin{tabular}{|c|c|c|c|c|c|c|c|}
\hline & \multicolumn{2}{|c|}{$\begin{array}{l}\text { Estadísticas } \\
\text { de muestras } \\
\text { emparejadas }\end{array}$} & \multicolumn{5}{|c|}{$\begin{array}{c}\text { Prueba t de dos muestras emparejadas } \\
\text { Variables ordinales (escalas) } \\
\text { Variables dicotómicas } \\
\text { (escala con punto de corte) }\end{array}$} \\
\hline & \multirow{3}{*}{$\begin{array}{l}\text { Media } \\
\text { Pre }\end{array}$} & \multirow{3}{*}{$\begin{array}{l}\text { Media } \\
\text { Post }\end{array}$} & \multicolumn{3}{|c|}{ Diferencias emparejadas } & \multirow[t]{3}{*}{$\mathbf{t}$} & \multirow[t]{3}{*}{ Sig. ${ }^{a}$} \\
\hline & & & \multirow[t]{2}{*}{ Media } & \multicolumn{2}{|c|}{$\begin{array}{l}95 \% \text { de intervalo } \\
\text { de confianza }\end{array}$} & & \\
\hline & & & & Inferior & Superior & & \\
\hline Índice Barthel & $\begin{array}{l}95,571 \\
(6,835)\end{array}$ & $\begin{array}{l}95,143 \\
(6,251)\end{array}$ & $\begin{array}{c}0,429 \\
(5,337)\end{array}$ & $-1,405$ & 2,262 & 0,475 & 0,638 \\
\hline Escala Lawton y Brody & $\begin{array}{l}7,457 \\
(1,268)\end{array}$ & $\begin{array}{c}7,657 \\
(0,938)\end{array}$ & $\begin{array}{l}-0,200 \\
(0,933)\end{array}$ & $-0,521$ & 0,121 & $-1,268$ & 0,213 \\
\hline MMSE Puntaje total corregido & $\begin{array}{l}26,943 \\
(2,890)\end{array}$ & $\begin{array}{l}26,914 \\
(3,212)\end{array}$ & $\begin{array}{c}0,029 \\
(2,162)\end{array}$ & $-0,714$ & 0,771 & 0,078 & 0,938 \\
\hline Yesavage & $\begin{array}{c}2,171 \\
(1,505)\end{array}$ & $\begin{array}{c}1,257 \\
(1,146)\end{array}$ & $\begin{array}{c}0,914 \\
(1,245)\end{array}$ & 0,486 & 1,342 & 4,343 & 0,000 \\
\hline Escala de soledad de HUGHES & $\begin{array}{c}5,457 \\
(2,227)\end{array}$ & $\begin{array}{c}5,143 \\
(1,700)\end{array}$ & $\begin{array}{c}0,314 \\
(1,711)\end{array}$ & $-0,273$ & 0,902 & 1,087 & 0,285 \\
\hline MSPSS Percepción apoyo social & $\begin{array}{c}34,029 \\
(10,706)\end{array}$ & $\begin{array}{c}37,286 \\
(10,368)\end{array}$ & $\begin{array}{l}-3,257 \\
(7,913)\end{array}$ & $-5,975$ & $-0,539$ & $-2,435$ & 0,020 \\
\hline Subtotal Fragilidad física & $\begin{array}{c}4,029 \\
(1,917)\end{array}$ & $\begin{array}{c}3,029 \\
(1,963)\end{array}$ & $\begin{array}{c}1,000 \\
(1,766)\end{array}$ & 0,393 & 1,607 & 3,351 & 0,002 \\
\hline Subtotal Fragilidad psicológica & $\begin{array}{c}2,057 \\
(1,027)\end{array}$ & $\begin{array}{c}1,829 \\
(0,857)\end{array}$ & $\begin{array}{c}0,229 \\
(1,031)\end{array}$ & $-0,126$ & 0,583 & 1,311 & 0,199 \\
\hline Subtotal Fragilidad social & $\begin{array}{c}1,086 \\
(0,887)\end{array}$ & $\begin{array}{c}1,029 \\
(0,785)\end{array}$ & $\begin{array}{c}0,057 \\
(0,639)\end{array}$ & $-0,162$ & 0,277 & 0,529 & 0,600 \\
\hline Puntaje Total fragilidad & $\begin{array}{c}7,143 \\
(3,098)\end{array}$ & $\begin{array}{c}6,029 \\
(2,595)\end{array}$ & $\begin{array}{c}1,114 \\
(2,336)\end{array}$ & 0,312 & 1,917 & 2,822 & 0,008 \\
\hline
\end{tabular}

aSignificación estadística bilateral. Entre paréntesis las respectivas desviaciones estándar de las medias. 
sión. En relación al área social, apoyo social medido por MSPSS reportó un promedio de 34,03 \pm 0,71; y la Escala de Soledad obtuvo un promedio de $5,46 \pm 2,23$. Finalmente, con respecto a estado de fragilidad, las PM reportaron un promedio de $7.14 \pm 3,10$ ), reportando $80,0 \%$ un puntaje de $5 \mathrm{o}$ más, clasificados como con fragilidad.

\section{Impacto en la VGI de las PM egresadas del $C D P M$}

Los resultados a los cambios en la funcionalidad se muestran en las Tablas 2 y 3 . Las PM no tuvieron cambios en la funcionalidad ni en las AVD ni en las AIVD entre los dos tiempos de evaluación. En relación al cambio en el área cognitiva y psicológica, se reportó un cambio estadísticamente significativo en el tamizaje de depresión, reportando menos puntaje al momento del egreso del CDPM. La escala de deterioro cognitivo no mostró cambios. Con respecto al área social, la escala de percepción de apoyo social reportó cambios entre la T1 y T2 de este estudio mostrando una mayor percepción de apoyo social las PM que egresaban del centro en comparación con su llegada al mismo. La escala de soledad no mostró cambios estadísticamente significativos.

\section{Impacto en la fragilidad de las PM egresadas del CDPM}

La mayoría de las PM disminuyó su grado de fragilidad después de 5 meses de asistencia a las intervenciones desarrolladas en el CDPM. La fragilidad total disminuyó significativamente entre el ingreso al centro (media $=7,143 \pm 3,10$ )

Tabla 3. Funcionalidad y fragilidad de las personas mayores asistentes al Centro de Día $(\mathbf{n}=35)$

\begin{tabular}{|c|c|c|c|c|}
\hline & $\begin{array}{l}\text { Variables } \\
\text { (ess } \\
\text { Prueba de } \\
\text { rangos con } \\
\text { signo de } \\
\text { Wilcoxon }\end{array}$ & $\begin{array}{l}\text { Pruebas no par } \\
\text { s ordinales } \\
\text { calas) }\end{array}$ & $\begin{array}{c}\text { aramétricas para muestras emparejadas } \\
\text { Variables dicotómicas } \\
\text { (escala con punto de corte) } \\
\% \text { escala dicotómica }\end{array}$ & $\begin{array}{l}\text { Prueba de } \\
\text { McNemar } \\
\text { Sig. }^{\text {a }}\end{array}$ \\
\hline & $\mathbf{Z}$ & $\begin{array}{c}\text { Antes de la } \\
\text { intervención } \\
\text { Sig. }{ }^{\text {a }}\end{array}$ & $\begin{array}{l}\text { Después de la } \\
\text { intervención }\end{array}$ & \\
\hline Índice Barthel &,- 528 & 0,597 & $\begin{array}{cc}62,9 \% & 60,0 \% \\
\text { (Dependencia leve/moderada } & <100 \text { puntos) }\end{array}$ & 1,000 \\
\hline Escala Lawton y Brody & $-1,265$ & 0,206 & $\begin{array}{lr}22,9 \% & 14,3 \% \\
\text { (Alguna dificultad }<8 \text { puntos) }\end{array}$ & 0,25 \\
\hline MMSE Puntaje total corregido & $-0,316$ & 0,752 & $\begin{array}{cc}8,6 \% & 8,6 \% \\
\text { (Tamizaje sugerencia de demencia }<24 \text { puntos) }\end{array}$ & 1 \\
\hline Yesavage & $-3,555$ & 0,000 & $\begin{array}{cc}62,9 \% & 31,4 \% \\
\text { (Sugerencia de depresión }<=2 \text { puntos) }\end{array}$ & 0,007 \\
\hline Escala de soledad de HUGHES & $-1,231$ & 0,218 & & \\
\hline MSPSS Percepción apoyo social & $-2,391$ & 0,017 & & \\
\hline Subtotal Fragilidad física & $-3,086$ & 0,002 & & \\
\hline Subtotal Fragilidad psicológica & $-1,269$ & 0,205 & & \\
\hline Subtotal Fragilidad social & $-0,535$ & 0,593 & & \\
\hline Puntaje Total fragilidad & $-2,782$ & 0,005 & & \\
\hline
\end{tabular}

aSignificación estadística bilateral. Entre paréntesis las respectivas desviaciones estándar de las medias. 
Tabla 4. Ítems fragilidad al ingreso y egreso del Centro de Día

\begin{tabular}{|c|c|c|c|c|c|}
\hline & & \multicolumn{3}{|c|}{$\begin{array}{c}\text { Estadísticas de muestras } \\
\text { emparejadas }\end{array}$} & \multirow{2}{*}{$\begin{array}{c}\text { Pruebas no paramétricas } \\
\text { para muestras emparejadas } \\
\text { (variable dicotómicas) } \\
\text { Prueba de McNemar } \\
\text { Significación exacta } \\
\text { (bilateral) }\end{array}$} \\
\hline & & $\mathbf{n}$ & $\begin{array}{c}\% \text { Antes } \\
\text { de la } \\
\text { intervención }\end{array}$ & $\begin{array}{c}\text { \% después } \\
\text { de la } \\
\text { intervención }\end{array}$ & \\
\hline $\begin{array}{l}\text { Fragilidad } \\
\text { física }\end{array}$ & $\begin{array}{l}\text { NO se siente saludable físicamente } \\
\text { sí Ha perdido peso últimamente sin } \\
\text { intención de hacerlo } \\
\text { sí tiene dificultad para caminar } \\
\text { sí tiene dificultad para mantener el } \\
\text { equilibrio } \\
\text { sí tiene mala audición } \\
\text { sí tiene mala visión } \\
\text { sí tiene falta de fuerza en las manos } \\
\text { sí tiene cansancio físico }\end{array}$ & $\begin{array}{l}35 \\
35 \\
35 \\
35 \\
35\end{array}$ & $\begin{array}{l}25,7 \% \\
20,0 \% \\
62,9 \% \\
\\
65,7 \% \\
34,3 \% \\
65,7 \% \\
54,3 \% \\
71,4 \%\end{array}$ & $\begin{array}{l}51,4 \% \\
25,7 \% \\
48,6 \% \\
45,7 \% \\
57,1 \%\end{array}$ & $\begin{array}{l}1,000 \\
1,000 \\
0,077 \\
0,125 \\
0,453 \\
0,180 \\
0,508 \\
0,180\end{array}$ \\
\hline $\begin{array}{l}\text { Fragilidad } \\
\text { psicológica }\end{array}$ & $\begin{array}{l}\text { SÍ/A VECES tiene problemas con su } \\
\text { memoria } \\
\text { Sí/A VECES se ha sentido con falta } \\
\text { de ánimo (bajoneado) durante el } \\
\text { último mes } \\
\text { sí/ A VECES se ha sentido nervioso o } \\
\text { ansioso durante el último mes } \\
\text { NO es capaz de enfrentar los proble- } \\
\text { mas de una buena forma }\end{array}$ & 35 & $\begin{array}{l}80,0 \% \\
80,0 \% \\
22,9 \%\end{array}$ & $82,9 \%$ & $\begin{array}{l}1,000 \\
1,000\end{array}$ \\
\hline $\begin{array}{l}\text { Fragilidad } \\
\text { social }\end{array}$ & $\begin{array}{l}\text { SÍ vive solo/a } \\
\text { Sí/A VECES echa de menos tener } \\
\text { gente alrededor suyo } \\
\text { NO recibe suficiente apoyo de otras } \\
\text { personas }\end{array}$ & 35 & $60,0 \%$ & $68,6 \%$ & 0,375 \\
\hline
\end{tabular}

y el egreso (media $=6,10 \pm 2,60$ ), con un intervalo de confianza para la diferencia de medias entre 0,312 y 1,917 . El test de Wilcoxon dio un resultado en la misma dirección ( $\mathrm{p} \pm 0,005)$. Si bien hubo disminución del promedio en cada uno de los tres dominios de fragilidad, sólo la disminución de la fragilidad física fue estadísticamente significativa tanto en el test $t$ de diferencia de medias pareadas (IC = 0,393-1,607), como en el Test de Wilcoxon $(\mathrm{p}=0,002)$.

En la Tabla 4 se muestran los cambios en los ítems de fragilidad de las PM. A pesar que en casi todas las variables hay una disminución del porcentaje de respuestas de sugerencia de fragilidad, en la Prueba McNemar sólo la percepción de que no recibe suficiente apoyo de otras personas disminuyó de $34,3 \%$ a $11,4 \%(\mathrm{p}=0,021)$ y 1 variable fue significativa a $90 \%$ : la dificultad para caminar disminuyó de 62,9\% a 40,0\% (p = 0,077).

\section{Discusión}

Este es el primer estudio que reporta cambios en la VGI y fragilidad de PM egresadas de CDPM de carácter social en Santiago, Chile. La población en estudio fue mayoritariamente de sexo femenino, soltera, con enseñanza básica, con buena autopercepción de salud, con al menos una enfermedad crónica. Asimismo, son personas con dependencia leve, sin dificultad para realizar sus AIVD, sin estado sugerente de demencia, con un alto porcentaje de tamizaje sugerente de depresión, y con un adecuado apoyo social percibido.

Ninguna de las mediciones de funcionalidad (AVD y AIVD) demostraron tener cambios estadísticamente significativos en este estudio. Una posible explicación para este hallazgo puede estar relacionado a que las actividades realizadas en el CDPM, permiten el desarrollo de herramientas 
para desenvolverse de una mejor forma en su vida diaria, pero no abordan directamente el desarrollo de ABVD/AIVD de las PM asistentes al CDPM.

Existieron cambios positivos en las $\mathrm{PM}$ al egreso del CDPM, en el tamizaje de depresión y en la percepción de apoyo social. Esto puede deberse a que las actividades desarrolladas tienen un fuerte componente grupal, participativo y social. A su vez, el centro cumple con la función de reconectar a las PM con las redes sociales disponibles en su comunidad, apoyando la percepción de apoyo social percibido.

Tanto la fragilidad total, como el componente físico por sí solo, demostraron tener cambios positivos para los mayores al egreso del CDPM. Las PM necesitan tener acceso rápido a servicios destinados a recibir una atención especializada que les permita mantener por mayor tiempo su autonomía ${ }^{26}$. En este sentido los CDPM se transforman en una oportunidad para contribuir a recuperar PM frágiles que viven en la comunidad.

Llama la atención el alto porcentaje de fragilidad encontrado. Este resultado puede deberse a que la muestra en estudio había sido referida al CDPM y, por ende, eran PM que requerían atención profesional. Por otro lado, el instrumento de fragilidad mide el concepto de forma multidimensional, incorporando la dimensión psicológica y social a la física. La fragilidad puede ser definida como: "un estado dinámico que afecta a un individuo que experimenta pérdidas en uno o más dominios del funcionamiento humano (físico, psicológico, social) que son causadas por la influencia de una serie de variables, y que aumenta el riesgo de resultados adversos" ${ }^{\prime 27}$. Esta definición ejemplifica la naturaleza multidimensional, dinámica y predictora de resultados adversos en salud del concepto ${ }^{28}$. No existe una definición única de fragilidad, lo que se puede plasmar en los distintos instrumentos de medición que existen para medirla, que varían desde considerar todos o algunos de los aspectos de la fragilidad física, cognitiva y/o social ${ }^{29-30}$. El concepto de fragilidad multidimensional desarrollado por Gobbens, Luijkx, Wijnen-Sponselee y Schols ${ }^{31}$ fue el usado en esta investigación.

Ninguna de las variables de funcionalidad y cognitivas tuvieron cambios estadísticamente significativos. Al ser estos servicios socio-sanitarios, por definición, no están orientados a que sean las variables de funcionales o cognitivas aquellas que cambien, sino que dan la apertura a la utilización de instrumentos menos tradicionales para la medición de sus resultados. Por ejemplo, la fragilidad desde una mirada multidimensional ${ }^{32}$ y la autoeficacia que es una de las variables que en Chile se ha encontrado más correlacionada con calidad de vida en la vejez ${ }^{33}$. Sería recomendable, entonces, incorporar este tipo de indicadores en futuras evaluaciones de los CDPM.

Este estudio tiene una serie de limitaciones. Primero, son PM atendidas en un solo CDPM, por lo tanto, no representan la variabilidad que pueden tener otros centros. Segundo, la muestra en estudio es mayoritariamente autovalente, sin diagnóstico de demencia y con un alto porcentaje de tamizaje de depresión, lo que no necesariamente representa la salud de la población general de $\mathrm{PM}$. Tercero, la muestra es pequeña y puede haber variables que no fueron significativas. Dentro de sus fortalezas está el tipo de diseño, longitudinal y muestra randomizada.

En conclusión, los CDPM mostraron en Chile ser una alternativa para aquellas $\mathrm{PM}$ que requieren apoyo de forma que disminuyen sus índices de fragilidad, aumentan su percepción de apoyo social y disminuyen su tamizaje de depresión a través de la asistencia regular a las actividades desarrolladas en este tipo de servicio geriátrico.

\section{Referencias}

1. Savard J, Leduc N, Lebel P, Béland F, Bergman H. Determinants of adult day center attendance among older adults with functional limitations. J Aging Health 2009; 21 (7): 985-1015.

2. Sviden GA, Tham K, Borell L. Elderly participants of social and rehabilitative day centres. Scand J Caring Sci 2004; 18(4): 402-9.

3. Dabelko HI, Balaswamy S. Use of adult day services and home health care services by older adults: A comparative analysis. Home Health Care Serv Q 2008; 18 (3): 65-79.

4. Baumgarten M, Lebel P, Laprise H, Leclerc C, Quinn C. Adult day care for the frail elderly: outcomes, satisfaction, and cost. J Aging Health 2002; 14 (2): 237-59.

5. OMS. Envejecimiento activo: Un marco político. Rev Esp Geriatr Gerontol 2002; 37 (2): 74-105.

6. Krout JA. Seniors centers and services for the frail elderly. J Aging Soc Policy 1995; 7 (2): 59-76.

7. Zank S, Schacke C. Evaluation of geriatric day care units: effects on patients and caregivers. J Gerontol B Psychol Sci Soc Sci 2002; 57 (4): P348-57. 
8. Ron P. Self-esteem among elderly people receiving care insurance at home and at day centers for the elderly. Int Psychogeriatr 2007; 19 (6): 1097-109.

9. Hashizume Y, Kanagawa K. Correlates of participation in adult day care and quality of life in ambulatory frail elderly in Japan. Public Health Nurs 1996; 13 (6): 40415.

10. Schmitt EM, Sands LP, Weiss S, Dowling G, Covinsky K. Adult day health center participation and health-related quality of life. Gerontologist 2010; 50 (4): 531-40.

11. Fitzpatrick TR, Gitelson RJ, Andereck KL, Mesbur ES. Social support factors and health among a senior center population in southern Ontario, Canada. Soc Work Health Care 2005; 40 (3): 15-37.

12. Iecovich E, Biderman A. Attendance in adult day care centers and its relation to loneliness among frail older adults. Int Psychogeriatr 2012; 24 (3): 439-48.

13. Warren S, Kerr JR, Smith D, Schalm C. The impact of adult day programs on family caregivers of elderly relatives. J Community Health Nurs 2003; 20 (4): 20921.

14. Cutchin MP. The process of mediated aging-in-place: a theoretically and empirically based model. Soc Sci Med 2003; 57 (6): 1077-90.

15. Kuzuya M, Masuda Y, Hirakawa Y, Iwata M, Enoki $\mathrm{H}$, Hasegawa J, et al. Day care service use is associated with lower mortality in community-dwelling frail older people. J Am Geriatr Soc 2006; 54 (9): 1364-71.

16. Mahoney FI, Barthel DW. Functional Evaluation: The Barthel Index. Md State Med J 1965; 14: 61-5.

17. Lawton MP, Brody EM. Assessment of older people: self-maintaining and instrumental activities of daily living. Gerontologist 1969; 9 (3): 179-86.

18. Folstein MF, Folstein SE, McHugh PR. "Mini-mental state". A practical method for grading the cognitive state of patients for the clinician. J Psychiatr Res 1975; 12 (3): 189-98.

19. Hoyl MT, Alessi CA, Harker JO, Josephson KR, Pietruszka FM, Koelfgen M, et al. Development and testing of a five-item version of the Geriatric Depression Scale. J Am Geriatr Soc 1999; 47 (7): 873-8.

20. Hughes ME, Waite LJ, Hawkley LC, Cacioppo JT. A Short Scale for Measuring Loneliness in Large Surveys: Results From Two Population-Based Studies. Res Aging 2004; 26 (6): 655-72.

21. Arechabala MC, Miranda C. Validación de una escala de apoyo social percibido en un grupo de adultos mayores adscritos a un porgrama de hipertensión arterial de la Rgeión Metropolitana. Cienc enferm 2002; 8 (1): 49-55.

22. Gobbens RJ, van Assen MA, Luijkx KG, Wijnen-Spon- selee MT, Schols JM. The Tilburg Frailty Indicator: psychometric properties. J Am Med Dir Assoc 2010; 11 (5): 344-55.

23. Santiago LM, Luz LL, Mattos IE, Gobbens RJ, van Assen MA. Psychometric properties of the Brazilian version of the Tilburg frailty indicator (TFI). Arch Gerontol Geriatr 2013; 57 (1): 39-45.

24. Daniels R, van Rossum E, Beurskens A, van den Heuvel $\mathrm{W}$, de Witte L. The predictive validity of three self-report screening instruments for identifying frail older people in the community. BMC Public Health 2012; 12: 69-2458-12-69.

25. Metzelthin SF, Daniels R, van Rossum E, de Witte L, van den Heuvel WJ, Kempen GI. The psychometric properties of three self-report screening instruments for identifying frail older people in the community. BMC Public Health 2010; 10: 176-2458-10-176.

26. Organización Mundial de la Salud. Acción multisectorial para un envejecimiento saludable basado en el ciclo de vida: proyecto de estrategia y plan de acción mundiales sobre el envejecimiento y la salud. [Internet]. 2016. [citado el 10 de septiembre de 2017]. Disponible en: http:// apps.who.int/gb/ebwha/pdf_files/WHA69/A69_17-sp. pdf?ua $=1$.

27. Gobbens R, Luijkx K, Wijnen-Sponselee M, Schols J. Toward a conceptual definition of frail community dwelling older people. Nurs Outlook 2010; 58: 76-86.

28. Sourial N, Wolfson C, Bergman H, Zhu B, Karunananthan S, Quail J, et al. A correspondence analysis revealed frailty deficits aggregate and are multidimensional. J Clin Epidemiol 2010; 63 (6): 647-54.

29. Sourial N, Bergman H, Karunananthan S, Wolfson C, Guralnik J, Payette H, et al. Contribution of frailty markers in explaining differences among individuals in five samples of older persons. J Gerontol A Biol Sci Med Sci 2012; 67 (11): 1197-204.

30. Woods NF, Cochrane BB, LaCroix AZ, Seguin RA, Zaslavsky $\mathrm{O}$, Liu J, et al. Toward a positive aging phenotype for older women: observations from the women's health initiative. J Gerontol A Biol Sci Med Sci 2012; 67 (11): 1191-6.

31. Gobbens RJ, Luijkx KG, Wijnen-Sponselee MT, Schols JM. Towards an integral conceptual model of frailty. J Nutr Health Aging 2010;14 (3): 175-81.

32. Iriarte E, Araya AX. Criterios de fragilidad en personas mayores que viven en la comunidad: una actualización de la literatura. Rev Med Chile 2016; 144: 1440-7.

33. Herrera MS, Barros C, Fernández MB. Predictors of Quality of Life in Old Age: A Multivariate Study in Chile. J Popul Ageing 2011; 4 (3): 121-39. 